17

\title{
Квантовое распределение ключей с временным кодированием tb-кубитов
}

\author{
(C) А.С. Задорин \\ Томский государственный университет систем управления и радиоэлектроники, \\ 634050 Томск, Россия \\ e-mail: Anatoly.Zadorin@gmail.com
}

Поступила в редакцию 16.11.2017 г.

В окончательной редакции 22.05.2018 г.

\begin{abstract}
Показана возможность реализации системы квантового распределения ключей (СКРК) с использованием временного кодирования tb-кубитов (time-bin qubit), приготавливаемых и измеряемых с помощью разбалансированных интерферометров Маха-Цендера. Показано, что для исключения четкой априорной идентификации tb-кубитов с равными базами их кодирование символами 0 и 1 может осуществляться за счет задержки одного из них на половину тактового интервала (ТИ). Показано, что однозначность измерений кутритов в данном алгоритме основывается на способности квантовых частиц в $\beta$-состояниях к интерференции амплитуд вероятностей в портах интерферометров системы, а также на отсутствии коллизий сигнальных состояний кутритов в смежных ТИ. Описан способ организации контроля таких коллизий в том числе за счет кубитов, приготавливаемых из квази-однофотонных когерентных лазерных состояний.
\end{abstract}

DOI: $10.21883 /$ OS.2018.09.46561.265-17

\section{Введение}

Развитие технологий квантовых вычислений во многом определяется прогрессом в разработке новых квантовых алгоритмов, успехами в адаптации их к различным форматам данных и существующей схемотехнике квантовых вентилей. Наглядным примером здесь могут служить разнообразные технологии построения систем квантового распределения ключей (СКРК), основанные на использовании квантовых процессоров, осуществляющих ряд логических операций над последовательностью кубитов $\left|\psi_{i}\right\rangle$. Иерархическая структура алгоритмов работы процессоров СКРК, как и других телекоммуникационных протоколов, содержит ряд уровней, объединенных в общий стек протоколов. Физический уровень в данной иерархии представлен протоколом генерации первичного ключа, включающим процедуры приготовления, измерения и обработки кубитов $\left|\psi_{i}\right\rangle$. Среди большого разнообразия алгоритмов генерации первичного ключа можно выделить несколько наиболее часто используемых базовых протоколов СКРК [1-4]. К их числу относятся такие классические протоколы, как ВВ84 1984 г. Ч. Беннета и Ж. Брассаро, В92 Ч. Беннета 1992 г. [5] и др., реализуемые в двух основных форматах кодирования - поляризационном и фазовом [1-6].

Наиболее простым в реализации оказывается вариант поляризационного кодирования $\left|\psi_{i}\right\rangle$. К сожалению, в оптоволоконных (ОВ) каналах связи поляризационные кубиты (ПК) испытывают сильную декогеренцию, т.е. быстро разрушаются вследствие поляризационной дисперсии ОВ. По этой причине основой большинства протоколов генерации первичного ключа в СКРК с ОВ в настоящее время является фазовое кодирова- ние кубитов [1-4]. Практическое использование данного метода затрудняется сложностью контроля состояния поляризации, а также случайных фазовых сдвигов в квантовом канале (КК) СКРК. Применение в СКРК автокомпенсационных схем лишь частично решает эту проблему. Так, система plug-and-play [6] позволяет устранить поляризационные искажения оптических сигналов за счет их двойного прохода по квантовому каналу с промежуточным отражением от зеркал Фарадея, однако вносит свои ограничения. К ним относятся снижение квантового битрейта, защищенной длины КК и др. [7].

В данной связи актуальной задачей развития технологической базы СКРК является отыскание и исследование альтернативных способов кодирования $\left|\psi_{i}\right\rangle$. В качестве одного из них можно рассматривать способ построения однокубитовых логических процессоров СКРК на основе временного кодирования tb-кубитов, ранее предложенный в [8] для построения первичного протокола ВВ84. Целью настоящей работы является обсуждение возможностей дальнейшего развития указанного подхода.

\section{Техника приготовления и измерения tb-кубитов}

В настоящее время схемотехника приготовления и измерения tb-кубитов хорошо разработана для систем КРК с фазовым кодированием [1-6]. Квантовый процессор этих систем содержит некоторый набор логических квантовых вентилей, распределенных между удаленными пользователями А и Б (ПА, ПБ), соединенными квантовым (ККС) и классическим каналами связи. Наиболее широкоиспользуемым в этих системах вентилем является разбалансированный интерферометр Маха- 
Цендера (ИМЦ), объединяющий несколько логических устройств: однокубитовые квантовые вентили Адамара, сдвигающий и фазовращающий вентили, реализованные в виде ОВ-линий задержки на время $\Delta$ и регулятора фазы $\alpha$ в плечах интерферометра $[4,9]$. В данной системе трансформация исходного когерентного состояния $\left|\psi_{0}\right\rangle$ квазиодиночного фотона в состояние $|\psi\rangle$ на выходе ИМЦ будет определяться последовательным произведением соответствующих унитарных операторов $\mathbf{H}, \mathbf{P}$ и D указанных квантовых вентилей. Для отыскания связи кет-векторов $\left|\psi_{0}\right\rangle$ и $\left|\psi_{4}\right\rangle$ вычислительный базис кубита и операторов $\mathbf{H}, \mathbf{P}$ и $\mathbf{D}$ удобно совместить с номерами портов интерферометра $|0\rangle,|1\rangle,|3\rangle$ и $|4\rangle$. В указанных координатах реакция ИМЦ $\left|\psi_{4}\right\rangle$, например, на возбуждение $\left|\psi_{0}\right\rangle=|0\rangle$, т.е. посылку фотона в порт $|0\rangle$, будет описываться соотношением [9],

$$
|\psi\rangle=\frac{1}{2}\left[e^{j \alpha_{0}} D+e^{j \alpha_{1}}\right]|3\rangle+\frac{1}{2}\left[e^{j \alpha_{0}} D-e^{j \alpha_{1}}\right]|4\rangle,
$$

где $D, \alpha_{0}, \alpha_{1}$ - унитарный оператор сдвига, описывающий относительное временное смещение одиночных фотонов на время $\Delta$, а также соответствующие фазовые сдвиги в плечах интерферометра.

Из последнего соотношения следует, что в портах $|3\rangle$ и $|4\rangle$ разбалансированного ИМЦ $|\psi\rangle$ представляется суперпозицией двух разделенных промежутком времени $\Delta$ состояний. Обозначим данные состояния кет-векторами $|\alpha\rangle$ и $|\beta\rangle$ и используем их для образования нового двумерного ортогонального динамического вычислительного базиса указанных квантовых объектов $\left|\psi_{i}\right\rangle$ :

$$
\left|\psi_{i}\right\rangle=\langle i \mid \psi\rangle=\xi_{i \alpha}|\alpha\rangle+\xi_{i \beta}|\beta\rangle,
$$

где $i=3,4 ; \xi_{j \alpha}, \xi_{i \beta}$ - комплексные амплитуды вероятности состояний $|\alpha\rangle$ и $|\beta\rangle$ объекта $\left|\psi_{i}\right\rangle$ в портах $|3\rangle$ и $|4\rangle$ соответственно. В литературе суперпозиция (2) называется time-bin qubit [9]. Ниже она обозначается как временной или tb-кубит. Временной сдвиг $\Delta$ между базисными состояниями $|\alpha\rangle$ и $|\beta\rangle$ будем называть базой ИМЦ.

Измерение временных кубитов $\left|\psi_{i}\right\rangle$ осуществляется с помощью второго интерферометра Б, аналогичного рассмотренному выше ИМЦ-А. Результат расчета кет-вектора $\left|\psi_{4}\right\rangle$ в системе из двух последовательно включенных интерферометров несложно получить путем замены состояния $\left|\psi_{0}\right\rangle=|0\rangle$ на входе ИМЦ-Б на соотношение (2). При этом следует учесть различие матриц $\mathbf{P}$ фазовращающих вентилей интерферометров. Эти различия в дальнейшем будем помечать нижними индексами фазовых переменных А и Б, например как $\alpha_{\mathrm{A} 0}$ или $\alpha_{\text {Б1 }}$. Кроме этого, обозначим операторы сдвига интерферометров как $D_{\mathrm{A}}$ и $D_{\mathrm{L}}$. В результате можно показать, что отклик $|\psi\rangle$ системы интерферометров, соединенных каналом из одного оптического волокна, на возбуждение $\left|\psi_{0}\right\rangle=|0\rangle$ имеет вид [9],

$$
\begin{aligned}
& |\psi\rangle=\frac{e^{j \alpha_{q c 0}}}{4}\left[e^{j\left(\alpha_{\mathrm{B} 0}+\alpha_{\mathrm{A} 0}\right)} D_{\mathrm{E}} D_{\mathrm{A}}+\left(e^{j\left(\alpha_{\mathrm{E} 0}+\alpha_{\mathrm{A} 1}\right.} D_{\mathrm{Б}}\right.\right. \\
& \left.\left.+e^{j\left(\alpha_{\mathrm{B} 1}+\alpha_{\mathrm{A} 0}\right.} D_{\mathrm{A}}\right)+e^{j\left(\alpha_{\mathrm{B} 1}+\alpha_{\mathrm{A} 1}\right)}\right]|3\rangle \\
& +\frac{e^{j \alpha_{q c 0}}}{4}\left[e^{j\left(\alpha_{\mathrm{B} 0}+\alpha_{\mathrm{A} 0}\right)} D_{\mathrm{Б}} D_{\mathrm{A}}\right)+\left(e^{j\left(\alpha_{50}+\alpha_{\mathrm{A} 1}\right)} D_{\mathrm{Б}}\right. \\
& \left.\left.-e^{j\left(\alpha_{51}+\alpha_{A 0}\right)} D_{\mathrm{A}}\right)-e^{j\left(\alpha_{\mathrm{B} 1}+\alpha_{\mathrm{A} 1}\right)}\right]|4\rangle .
\end{aligned}
$$

Из (3) следует, что в каждом из портов $|3\rangle$ и $|4\rangle$ ИМЦ-Б объект $\left|\psi_{4}\right\rangle$ в общем случае представлен суперпозицией из четырех динамических состояний. Одно из них является состоянием с нулевой задержкой $(D=1)$, реализуемым на оптической траектории $\mathrm{K}_{\mathrm{A}}-\mathrm{K}_{\mathrm{b}}$, еще два состояния с однократной задержкой $\left(D=D_{\mathrm{A}}, D_{\mathrm{E}}\right)$, реализуемые на траекториях ДА $-K_{\mathrm{L}}$ и $\mathrm{K}_{\mathrm{A}}-Д_{\mathrm{E}}$, а также одно состояние с двукратной задержкой $\left(D=D_{\mathrm{A}} \cdot D_{\text {Б }}\right)$ на траектории Д ${ }_{\mathrm{A}}-Д_{\mathrm{E}}$. При равных базах интерферометров, когда $D_{\mathrm{A}}=D_{\text {Б}}$, размерность вектора $|\psi\rangle$ снижается до 3 , т. е. $|\psi\rangle$ обращается в кутрит $|\varphi\rangle$, представленный тремя линейно независимыми динамическими состояниями, разнесенными по времени друг от друга на базовую задержку ИМЦ $\Delta$. Выберем указанные состояния в качестве базисных векторов объекта $|\varphi\rangle$ и обозначим как $|\alpha\rangle$, $|\beta\rangle$ и $|\gamma\rangle$. Далее, находим проекции $|\psi\rangle$ на векторы $|3\rangle$ и $|4\rangle$, определяющие вероятности регистрации фотона в выходных портах второго ИМЦ-Б,

$$
\left.\left.\left|\varphi_{i}\right\rangle=\langle i|\right] p s i\right\rangle=\xi_{i \alpha}|\alpha\rangle+\xi_{i \beta}|\beta\rangle+\xi_{i \gamma}|\gamma\rangle,
$$

где $i=3,4 ; \quad \xi_{i \alpha}, \xi_{i \beta}, \xi_{i \gamma}-$ комплексные амплитуды вероятности состояний $|\alpha\rangle$, $|\beta\rangle$ и $|\gamma\rangle$ кутрита $\left|\varphi_{i}\right\rangle$ в портах $|3\rangle$ и $|4\rangle$ соответственно.

Отметим, что состояние $|\beta\rangle$ является наиболее важным информационным состоянием кутрита (4), в котором у квантовой частицы появляется возможность интерферировать сама с собой в форме интерференции амплитуд вероятностей [11]. В этом случае при сдвиге фазы на траекториях $Д_{A}-K_{\mathrm{E}}$ и $\mathrm{K}_{\mathrm{A}}-Д_{\mathrm{E}}$, равном $\phi$, вероятности регистрации одиночных фотонов в портах $|3\rangle$ и $|4\rangle$ ИМЦ-Б будут [2]

$$
P_{1} \sim \cos ^{2}(\phi / 2) \text { и } P_{2} \sim \sin ^{2}(\phi / 2) .
$$

Соотношения (4), (5) являются формальной основой техники фазового кодирования и измерения кубитов $\left|\psi_{i}\right\rangle$ в ИМЦ, характерной особенностью которой является фиксированное положение векторов $|\alpha\rangle,|\beta\rangle$ и $|\gamma\rangle$ относительно границ тактового интервала. Естественная для данных квантовых объектов динамическая степень свободы здесь остается не востребованной. Ниже рассмотрим способ ее использования для расширения функциональных возможностей фазового кодирования tb-кубитов и построения на его основе протокола СКРК. 


\section{Временное кодирование tb-кубитов}

При временном кодировании каждый из двоичных символов связывается с различным положением или конфигурацией (относительными сдвигами базисных состояний в (2) и (3)) сигнального квантового объекта на $i$-м тактовом интервале $T_{i}$ (ТИ). Для $\left|\psi_{i}\right\rangle$ и $\left|\varphi_{i}\right\rangle$ в качестве сигнальных признаков можно использовать базу $\Delta$ ИМЦ и временную задержку $\tau$, измеряемую относительно начала ТИ. В дальнейшем изменения динамической структуры $\left|\psi_{i}\right\rangle$ и $\left|\varphi_{i}\right\rangle$, связанные с передаваемыми символами 0 и 1 , будем описывать зависимостью указанных объектов от дискретного параметра $l=0,1$ как $\mid \psi_{i}(l)$ и $\left|\varphi_{i}(l)\right\rangle$.

Основными критериями корректного выбора $\Delta$ и $\tau$ в СКРК для каждой конфигурации состояний $|\alpha\rangle,|\beta\rangle$ и $|\gamma\rangle$ объектов $\left|\psi_{i}(l)\right\rangle$ и $\left|\varphi_{i}(l)\right\rangle$ являются условия выполнения теоремы о запрете клонировния $[1,2]$, исключающие возможность четкой априорной идентификации указанных объектов с альтернативными символами $l$. При отыскании этих условий следует иметь в виду, что представление $\left|\psi_{i}(l)\right\rangle$ и $\left|\varphi_{i}(l)\right\rangle$ в виде суперпозиции базисных состояний (2) сохраняется лишь до акта измерения. В момент измерения волновая функция кубита с вероятностью, определяемой комплексной амплитудой $\xi_{i j}$, переходит в одно из базисных состояний $[1,2,11]$.

Возможными конфигурациями tb-объектов $\left|\psi_{i}(l)\right\rangle$ и $\left|\varphi_{i}(l)\right\rangle$, удовлетворяющими указанным условиям, представляются структуры на рис. 1. На этом рисунке внутри каждого $i$-го ТИ штриховкой обозначены таймслоты $(\mathrm{TC}) \Delta t_{i}(0)$ и $\Delta t_{i}(1)$, представляющие собой сигнальные интервалы, в которых возможна локализация квази-одиночных фотонов из базисных состояний $|\alpha\rangle$, $|\beta\rangle$ и $|\gamma\rangle$ объектов $\psi(l)\rangle$ и $|\varphi(l)\rangle$. Из рисунка видно, что основной особенностью данных динамических структур является то, что ТС тактовых интервалов раз- решены для появления в них нескольких различных состояний tb-кубитов и кутритов $\left|\psi_{i}(l)\right\rangle$ и $\left|\varphi_{i}(l)\right\rangle$ из смежных ТИ. Такое наложение tb-объектов является признаком их динамической неортогональности и может служить основой для построения соответствующего протокола СКРК. Препятствием для этого является наличие в тайм-слоте $\Delta t_{i}(l)$ разрешенных состояний равновероятных квантовых объектов, ассоциированных с альтернативными символами 0 и 1 ключевого кода.

Такое ограничение относится, например, к кубитам $|\psi(0)\rangle$ и $|\psi(1)\rangle$, которые на рис. 1 характеризуются одинаковыми базами $\Delta=T / 2$ и различаются лишь задержкой $\tau=\Delta=T / 2$ относительно границы ТИ. Из рисунка видно, что в показанных структурах $\left|\psi_{i}(l)\right\rangle$ одиночный фотон может находиться в середине или по краям ТИ, в тайм-слотах $\Delta t_{i}(0), \Delta t_{i}(1)$ или $\Delta t_{i+1}(0)$. При этом если $\left|\xi_{i \alpha}\right|=\left|\xi_{i \beta}\right|$, то вероятности регистрации фотона в состояниях 0 и 1 в каждом из указанных ТС совпадают. В данных условиях четкое разделение в измеряемой последовательности $\left|\psi_{i}(l)\right\rangle$ кубитов на $|\psi(0)\rangle$ и $|\psi(1)\rangle$ не представляется возможным.

\begin{tabular}{l|c|c|c|c}
\hline \multirow{2}{*}{$P\left(\left|\varphi_{i}(l)\right\rangle\right)$} & \multicolumn{2}{|c|}{$t=\Delta t_{i}(0)$} & \multicolumn{2}{c}{$t=\Delta t_{i}(1)$} \\
\cline { 2 - 5 } & порт $|3\rangle$ & порт $|4\rangle$ & порт $|3\rangle$ & порт $|4\rangle$ \\
\hline$P\left(\left|\alpha_{i}(0)\right\rangle\right)$ & 0.125 & 0.125 & 0 & 0 \\
$P\left(\left|\gamma_{i-1}(0)\right\rangle\right)$ & 0.125 & 0.125 & 0 & 0 \\
$P\left(\left|\beta_{i}(0)\right\rangle\right)$ & 0 & 0 & 0 & 0.5 \\
$P\left(\left|\alpha_{i}(1)\right\rangle\right)$ & 0 & 0 & 0.125 & 0.125 \\
$P\left(\left|\gamma_{i-1}(1)\right\rangle\right)$ & 0 & 0 & 0.125 & 0.125 \\
$P\left(\left|\beta_{i-1}(1)\right\rangle\right)$ & 0 & 0.5 & 0 & 0
\end{tabular}

Воспользуемся данными рис. 1 для исследования возможности построения протокола СКРК на основе обработки tb-кутритов. В данном случае в каждом TC $\Delta t_{i}(l)$ разрешенным оказывается любое из трех состояний кутрита (4). При этом, во-первых, в силу вырожденности $\beta$-состояния $\left|\varphi_{i}(l)\right\rangle$ вероятность его регистрации равна сумме соответствующих вероятностей $\alpha$ - и $\gamma$-состояний, и, во-вторых, переносимый им кодовый символ всегда альтернативен символу, связанному с остальными векторами кутрита (4). Это значит, что в общем случае ПБ не в состоянии надежно разделить кодовые состояния $\left|\psi_{i}(l)\right\rangle$.

Для того чтобы исключить вероятность появления в ТC $\Delta t_{i}(l)$ состояний tb-кутрита с различными кодовыми символами, можно воспользоваться упомянутой ранее способностью квантовой частицы в $\beta$-состояниях к интерференции амплитуд вероятностей [11]. С этой целью в состав одного или обоих интерферометров ИМЦ-А, Б на рис. 2 вводятся фазовращающие вентили, обеспечивающие фазовые сдвиги $\phi_{\mathrm{A}}$ и $\phi_{\text {Б }}$ оптического сигнала в их плечах так, чтобы суммарный сдвиг был равен $\pi$, т.е.

$$
\phi_{\mathrm{A}}+\phi_{\mathrm{Б}}=\pi
$$

Подставим (6) в (5) полагая, что $\left|\xi_{i \alpha}\right|=\left|\xi_{i \beta}\right|$. Заключаем, что при посылке кубита $\left|\psi_{i}(l)\right\rangle$ в порт $|0\rangle$ ИМЦ-Б вследствие квантовой интерференции $\beta$-состояний tb-кутрита вероятности их регистрации в портах $|3\rangle$ и $|4\rangle$ (сигнальном и контрольном) ИМЦ-Б будут равны 0 и 0.5 соответственно. Вероятности $P\left(\left|\varphi_{i}(l)\right\rangle\right)$ для указанных и других состояний $\left|\varphi_{i}(l)\right\rangle$ в обоих портах ИМЦ-Б и тайм-слотах $\Delta t_{i}(l) i$-го ТИ для $\left|\xi_{i \alpha}\right|=\left|\xi_{i \beta}\right|$ приведены в таблице. В соответствии с этими данными, а также рис. 1, в условиях (6) в сигнальном порте $|3\rangle$ интерферометра Б разрешенными оказываются только $\alpha$ и $\gamma$-состояния tb-кутритов, локализованные в смежных $(i-1)$-м и $i$-м ТИ, но связанные с одинаковыми кодовыми символами $l=0,1$. Последнее означает возможность надежного разделения предаваемых символов.

Действительно, согласно изложенному, регистрация одиночного фотона в порте $|3\rangle$ ИМЦ-Б в момент ТС $\Delta t_{i}(0)$ означает передачу по квантовому каналу в $(i-1)$-м или $i$-м ТИ символа 0 , а регистрация в момент ТC $\Delta t_{i}(1)$ - символа 1 (на рис. 1 эти состояния кутритов $\left|\varphi_{i}(l)\right\rangle$ обведены пунктирной линией). Важно подчеркнуть, что динамическая неортогональность 


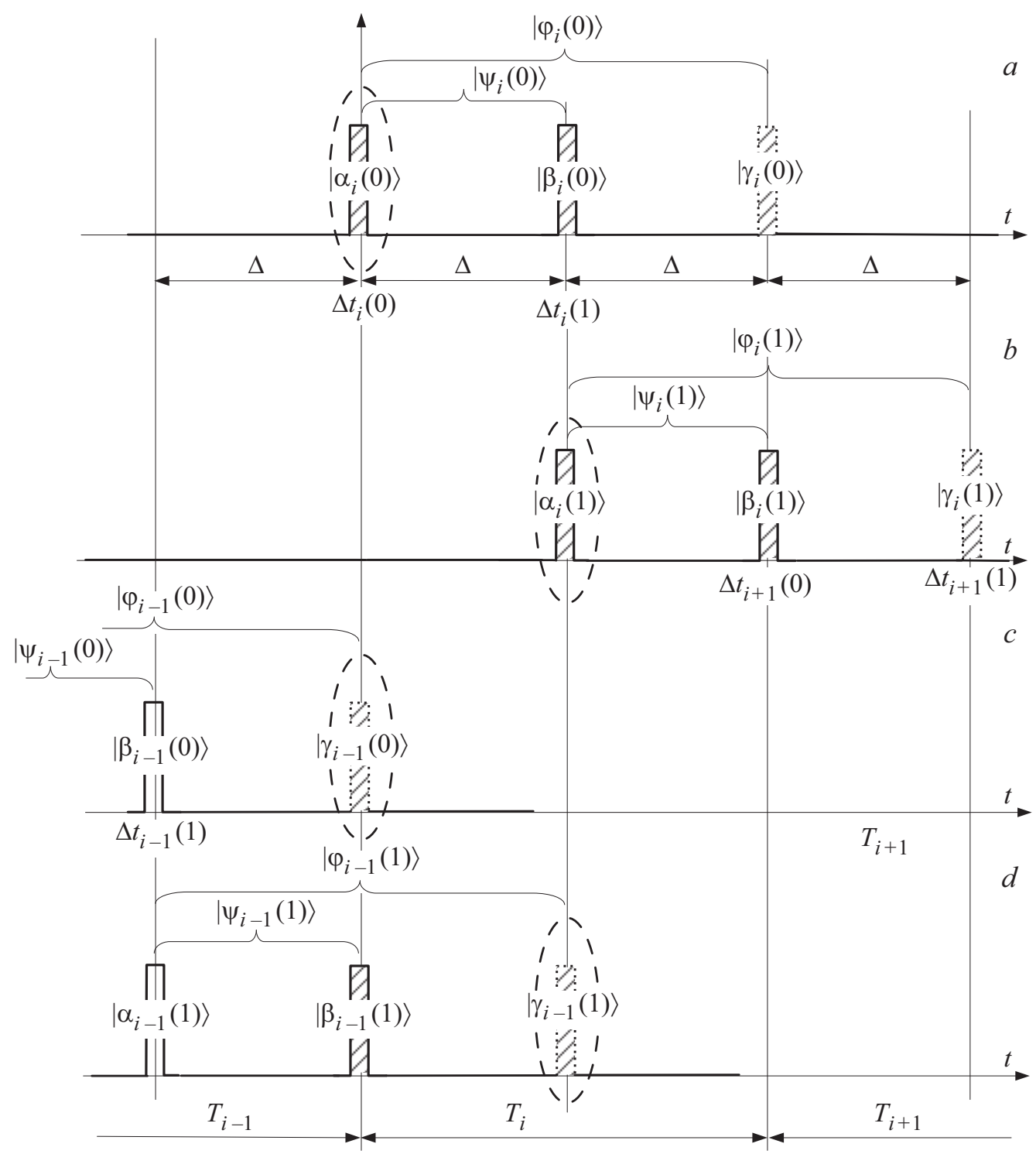

Рис. 1. Кодовые состояния кубитов $\left|\psi_{i}(l)\right\rangle$ и кутритов $\left|\varphi_{i}(l)\right\rangle$ при различных временных сдвигах относительно границ $i$-го ТИ (a) $\left.\left|\psi_{i}(0)\right\rangle,\left|\varphi_{i}(0)\right\rangle ;(b)\left|\psi_{i}(1)\right\rangle,\left|\varphi_{i}(1)\right\rangle ;(c)\left|\psi_{i-1}(0)\right\rangle,\left|\varphi_{i-1}(0) ;(d)\right| \psi_{i-1}(1)\right\rangle,\left|\varphi_{i-1}(1)\right\rangle$.

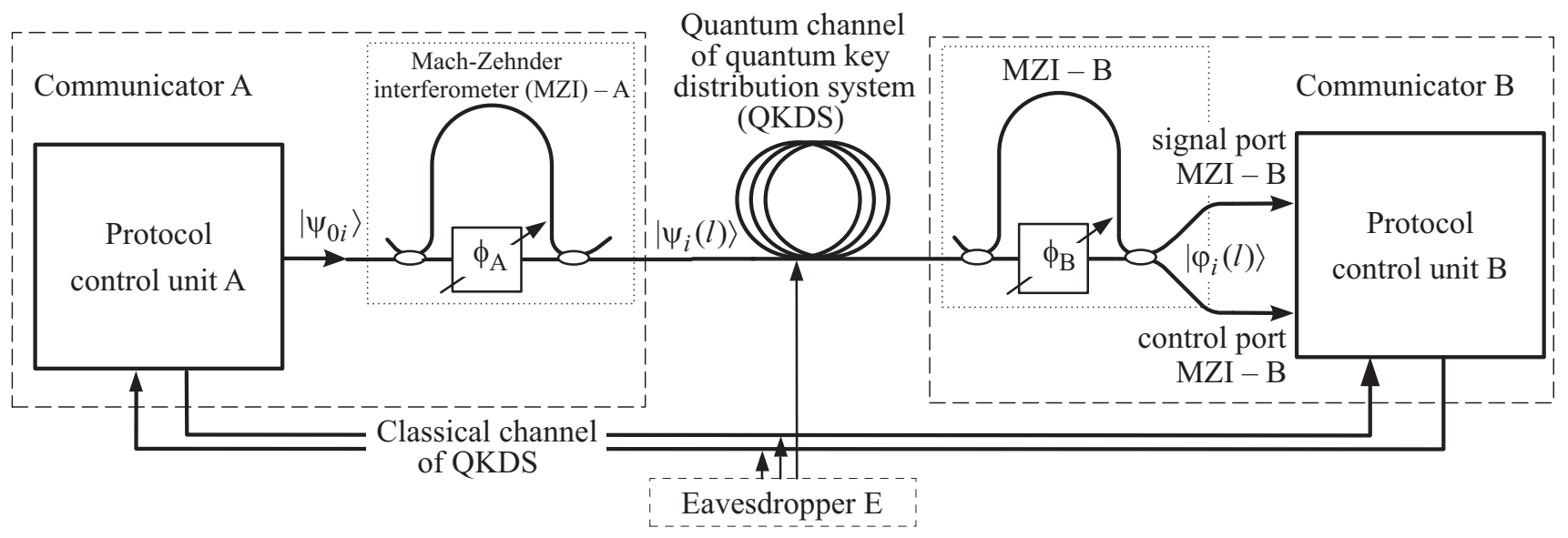

Рис. 2. Структурная схема СКРК с временным кодированием tb-кубитов. 
состояний объектов $\left|\varphi_{i}(l)\right\rangle$ здесь проявляется не в неопределенности значения символа $l$, а в его номере в последовательности $\left|\varphi_{i}(l)\right\rangle$. В рассматриваемом протоколе эту неопределенность и предлагается использовать для обеспечения основной защиты СКРК от попыток клонирования одиночных фотонов в квантовом канале нелегитимным пользователем (агентом Е (AE)).

Механизм защиты здесь может быть аналогичен, например, механизму защиты протокола В92 $[1,2,6]$. С этой целью на стороне ПА формируется случайная двоичная последовательность $\mathbf{m}_{\mathrm{A}}$ с числом элементов, равным числу тактовых интервалов во фрейме СКРК. Каждый элемент $\mathbf{m}_{\mathrm{A}}$ содержит два поля - поле номера элемента $i$ и поле кодового символа $m_{\mathrm{A} i}$. Элементы $m_{\mathrm{A} i}$ ПА использует для случайной смены значений кодовых символов $l$ в $i$-х ТИ последовательности кубитов $\left|\psi_{i}(l)\right\rangle$, приготовляемых в вычислительных базисах по рис. 1 , т. е.

$$
\left|\psi_{i}(l)\right\rangle=\left|\psi_{i}\left(m_{\mathrm{A} i}\right)\right\rangle
$$

Для исключения неоднозначных измерений последовательности $\left|\psi_{i}(l)\right\rangle$ при формировании $\mathbf{m}_{\mathrm{A}}$ стороной $\mathrm{A}$ не допускается возникновение коллизий, т. е наложений нескольких состояний кубитов из соседних тактовых интервалов в любом из его тайм-слотов. С этой целью поля кодовых символов в $\mathbf{m}_{\mathrm{A}}$ перемежаются случайным числом из нескольких (от 1-2) холостых полей, исключающих какую-либо регулярность в чередовании $\left|\psi_{i}(l)\right\rangle$ по параметру $l$. Такие вставки практически легко осуществимы, например, путем приготовления состояний $\left|\psi_{0}\right\rangle$ за счет ослабления лазерных импульсов до уровня, характеризуемого заданным средним числом фотонов в ТС $m \approx 0.1$ [1-7]. В данном случае вероятность обнаружить $n$ фотонов в состоянии $\left|\psi_{0}\right\rangle$ описывается пуассоновской статистикой, параметр $m$ которой определяет среднее число $\left(\sim m^{-1}\right)$ холостых полей между активными состояниями $\left|\psi_{0}\right\rangle$.

Сторона Б, анализируя далее состояния $\left|\psi_{i}(l)\right\rangle$ и $\left|\varphi_{i}(l)\right\rangle$, пытается определить некоторые элементы $\mathbf{m}_{\mathrm{A}}$ с целью создания на их основе ключевых кодовых последовательностей $\mathbf{k}_{\text {АБ. }}$ В условиях (6) и в отсутствие шумов эти попытки ПБ основаны на упомянутой выше возможности безошибочного определения кодового символа $l$ в зарегистрированном кутрите (рис. 1). Таким образом, проблема отыскания ПБ элементов $\mathbf{m}_{\mathrm{A} i}$ оказывается связанной только с восстановлением нумерации полей $m_{\mathrm{A} i}$. Для решения этой задачи ПБ формирует у себя собственную случайную двоичную последовательность $\mathbf{m}_{\text {Б }}$ с числом элементов, равным $\mathbf{m}_{\mathrm{A}}$, которая используется им для принятия решения о том, к какому $(i-1)$-му или $i$-му ТИ относится зарегистрированный в порте $|3\rangle$ ИМЦ-Б кутрит $\left|\varphi_{i}(l)\right\rangle$, или, иначе, какой номер $i$ в последовательности $\mathbf{m}_{\mathrm{A}}$ имеет принятый в $j$-м ТИ кодовый символ $l$. Решение ПБ о принятии той или иной гипотезы относительно номера $i$ можно представить, например, так:

$$
i=j-1+m_{\mathrm{b} j}
$$

где $m_{\mathrm{Б} j}-$ значение $j$-го двоичного символа (0 или 1) последовательности $\mathbf{m}_{\mathrm{b}}$.

В силу стохастичности $\mathbf{m}_{Б}$ данное решение приблизительно в половине случаев будет давать неправильный результат. В этой связи для использования восстановленных стороной Б фрагментов $\mathbf{m}_{\mathrm{A}}$ в качестве

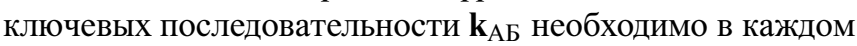
$i$-м ТИ предусмотреть процедуру устранения ошибок в решениях (8) (проверку состоятельности гипотез (8)), которая может производиться следующим образом. При регистрации в сигнальном порте $|3\rangle$ ИМЦ-Б $j$-го ТИ состояния пользователь Б по классическому каналу СКРК (рис. 2) сообщает стороне А текущее значение элемента $m_{\text {Б }}$ в формуле (8), не раскрывая при этом значение самого кодового символа $l$. В ответ ПА подтверждает или не подтверждает правильность такого решения. Указанная квитанция является основанием для включения ПБ очередных элементов $m_{\mathrm{A} i}$ в качестве

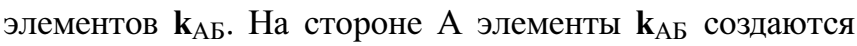
из $\mathbf{m}_{\mathrm{A}}$ за счет исключения из нее неактивных ТИ, т.е. тактовых интервалов, в которых не зарегистрированы $\alpha$ - или $\gamma$-состояния tb-кутритов в сигнальном порте $|3\rangle$ ИМЦ-Б. Исключаются также и поля ТИ, если зарегистрированные в них сигналы не прошли указанной выше проверки состоятельности.

В соответствии с приведенным описанием ключевая

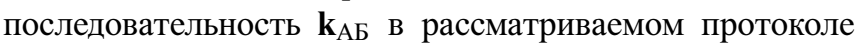
представляет собой согласованный сторонами набор фрагментов последовательности $\mathbf{m}_{\text {A }}$, элементы которой состоят из поля номера элемента $N_{i}$ и собственно кодового символа $l_{i}$. Совокупность всех значений $l_{i}$ в $\mathbf{k}_{\mathrm{AБ}}$ представляет собой генерируемый системой секретный код. Последовательность же $N_{i}$ секретной не является. Она вместе с приведенной в таблице статистикой распределений состояний кутритов $\left|\varphi_{i}(l)\right\rangle$ по портам ИМЦ-Б и тайм-слотам $\Delta t_{i}(l)$ ТИ представляет защитную информацию системы, предназначенную для детектирования попыток АЕ клонирования tb-кубитов в канале СКРК. Рассмотрим возможности использования этой информации при различных сценариях поведения АЕ.

Прежде всего отметим бесперспективность попыток $\mathrm{AE}$ подмены $\alpha$ - или $\beta$-состояний кубитов одиночными фотонами $\left|\psi_{0}\right\rangle$ и последующего анализа движения квитанций ПА в классическом канале с целью определения согласованных легитимными сторонами элементов $\mathbf{k}_{\text {АБ. }}$ Такое нарушение целостности $\left|\psi_{i}(l)\right\rangle$ приведет к разрушению интерференции амплитуд вероятностей этих квантовых объектов и, как следствие, наложению альтернативных символов в сигнальном порте ИМЦ-Б. Согласно данным таблицы, в этом случае в обоих портах этого интерферометра соответствующие вероятности будут одинаковы, т. е. $P(|\alpha(0,1)\rangle)+P(|\gamma(0,1)\rangle) \approx P(|\beta(1,0)\rangle)$. 
Последнее означает недопустимо высокий уровень системного показателя - коэффициента квантовых ошибок Q-BER.

Проанализируем далее возможности АЕ клонирования кубитов $\left|\psi_{i}(l)\right\rangle$ в квантовом канале СКРК (рис. 2). Для этой цели АЕ может использовать аппаратуру, аналогичную упомянутой выше аппаратуре легитимных пользователей. При такой попытке сторона $\mathrm{AE}$, как и законный пользователь Б, столкнется с необходимостью принятия решения типа (8) относительно номера ТИ, в котором должен быть приготовлен фальшивый кубит $\mid \psi_{i}(l)$, взамен зарегистрированного им $\mid \psi_{i}(l)$. Как рассматриваемый, так и другие известные протоколы СКРК исключает для АЕ возможность такой проверки. Поэтому неизбежные в таком случае ошибки в полях номера элемента $N_{i}$ формируемой на сторонах $\mathrm{A}$ и Б ключевой последовательности $\mathbf{k}_{\text {АБ}}$, связанные с появлением в канале СКРК кубитов $\left|\psi_{i}(l)\right\rangle$, позволяют детектировать указанную активность АЕ.

\section{Заключение}

Приведенное выше обсуждение указывает на возможность построения алгоритма временного кодирования tb-кубитов, а также соответствующих однокубитовых логических процессоров СКРК на этой основе. Показано, что однозначность измерений кутритов в данном алгоритме основывается на способности квантовых частиц в $\beta$-состояниях к интерференции амплитуд вероятностей в портах интерферометров системы, а также отсутствии коллизий сигнальных состояний кутритов в смежных ТИ. Описан способ организации контроля таких коллизий в том числе за счет кубитов, приготавливаемых из квазиоднофотонных когерентных лазерных состояний.

Следует отметить, что за рамками обсуждения остались такие вопросы, как оценка допустимого уровня коэффициента квантовых ошибок Q-BER и $B_{\kappa}$ - скорости формирования $\mathbf{k}_{\mathrm{Ab}}$ (битрейт), значения которых также используются для детектирования несанкционированных вторжений АЕ в систему [1-8]. По мнению автора, данные вопросы заслуживают отдельного обсуждения.

\section{Список литературы}

[1] Нильсен М., Чанг И. Квантовые вычисления и квантовая информация. М.: Мир, 2008. 824 с.

[2] Имре Ш., Балажс Ф. Квантовые вычисления и связь. Инженерный подход. М.: Физматлит, 2008. 320 с.

[3] Кулик С.П., Молотков С.Н., Маккавеев А.П. // Письма в ЖЭТФ, 2007. Т. 85. № 6. С. 354-359.

[4] Кронберг Д.А., Ожсигов Ю.И., Чернявский А.Ю. Квантовая криптография. Учебно-методическое пособие. М.: МаксПресс, 2011. 112 c.

[5] Zbinden H., Gautier J.D., Gisin N., Huttner B., Muller A., Tittel W. // Electron. Lett. 1997. V. 33. P. 586-588.

[6] Bennett C.H. // Phys. Rev. Lett. 1992. V. 68. P. 3121.
[7] Румянцев К.Е., Плёнкин А.П. // Радиотехника. 2015. T. № 2. C. $125-134$.

[8] Задорин А.С., Махорин Д.А. // Изв. вузов. Физика. 2016. T. 59. № 3. C. 24-29.

[9] Задорин А.С., Махорин Д.А. // Доклады ТУСУРа. 2015. T. 37. № 3. C. $145-149$.

[10] Gisin N., Ribordy G., Wolfgang T. // Rev. Mod. Phys. 2002. V. 74. P. $145-195$

[11] Дирак П. Принципы квантовой механики. М.: Наука, 1979. $480 \mathrm{c}$. 